\title{
INFLUENCIA DE LA DISTANCIA ENTRE LA CRESTA ÓSEA Y EL PUNTO DE CONTACTO INTERDENTAL EN LA FORMACIÓN DE PAPILAS GINGIVALES: ESTUDIO EN PERROS
}

\author{
${ }^{1}$ Diogo Rodrigues Cruvinel, ${ }^{2}$ Carla Cecilia Alandia-Román, ${ }^{3}$ Camilo Andrés Villabona López, \\ ${ }^{4}$ Valdir Antonio Muglia \\ ${ }^{1}$ Doctor en Rehabilitación Oral F. de Odontología U. de São Paulo Ribeirão Preto, Brasil. \\ ${ }^{2}$ Candidato a Doctor en Rehabilitación Oral F. de Odontología U. de São Paulo Ribeirão Preto, Brasil. \\ ${ }^{3}$ Magister en Reabilitação Oral F. de Odontología U. de São Paulo Ribeirão Preto, \\ candidato a Doctor en Implantologia U. Federal de Santa Catarina Florianópolis, Brasil, \\ Docente F. de Odontología U. Santo Tomás, Colombia. \\ ${ }^{4}$ Profesor Titular Departamento de Materiales Dentarios y Prótesis F. de Odontología U. de São Paulo Ribeirão Preto, Brasil.
}

Autor responsable de correspondencia: Camilo Andrés Villabona López

Correo electrónico: lacho.villa@hotmail.com

\begin{abstract}
RESUMEN
Objetivo: evaluar la influencia de la distancia entre la cresta ósea y el punto de contacto interdental ( 3 y 5 mm) y la distancia entre implantes (2 y $3 \mathrm{~mm}$ ) en la formación de papilas gingivales.

Materiales y métodos: ocho perros adultos jóvenes fueron seleccionados para el estudio. Se realizaron las exodoncias de los cuatro premolares inferiores de cada hemiarcada mandibular y después de tres meses se colocaron tres implantes dentales (4,3 x $10 \mathrm{~mm})$ en cada hemiarcada. El primer implante fue puesto a $10 \mathrm{~mm}$ del canino remanente, el segundo (intermedio) fue colocado a $2 \mathrm{~mm}$ del primero y el tercero a $3 \mathrm{~mm}$ del segundo. Las coronas metálicas definitivas fueron confeccionadas con distancias de $3 \mathrm{y} 5 \mathrm{~mm}$ entre la cresta ósea y el punto de contacto interdental. Se evaluaron clínicamente doce semanas después de la instalación de las prótesis para medir el espacio formado entre la papila y el punto de contaco y luego estos datos fueron analizados estadísticamente mediante el Análisis de Varianza (ANOVA) de dos vías y la prueba de Bonferroni. Se consideró un valor de $\mathrm{p}<0,05$ como estadísticamente significativo. Resultados: se encontró un espacio mayor en los lugares en que la distancia entre implantes era de 2 mm y la altura del punto de contacto de $5 \mathrm{~mm}$. El menor se obsevó en los sitios en que la distancia entre implantes era de $2 \mathrm{~mm}$ y altura del punto de contacto $3 \mathrm{~mm}$. Conclusión: los puntos de contacto de $3 \mathrm{~mm}$ influyen positivamente en la formación de las papilas gingivales. [Cruvinel DR, Alandia-Román CC, Villabona CA, Muglia VA. Influencia de la distancia entre la cresta ósea y el punto de contacto interdental en la formación de papilas gingivales: estudio en perros. Ustasalud 2013; 12: 101 - 107]
\end{abstract}

Palabras clave: Encía, Resorción ósea, Prótesis dental.

\section{DISTANCE INFLUENCE BETWEEN THE INTERDENTAL BONE CREST AND THE CONTACT POINT IN THE FORMATION OF GINGIVAL PAPILLA: STUDY IN DOGS}

\begin{abstract}
Objective: to evaluate the influence of the distance between the bone crest and the interdental contact point ( 3 and $5 \mathrm{~mm}$ ) and the distance between implants ( 2 and $3 \mathrm{~mm}$ ) in the formation of gingival papillae.

Methods: eight young adult dogs were selected for the study. Extractions of the four premolars of each hemi mandibular arch were performed and after three months three implants were placed $(4.3 \times 10 \mathrm{~mm})$ in each hemi arch. The first implant was placed $10 \mathrm{~mm}$ from the remnant canine, the second implant (intermediate) was placed $2 \mathrm{~mm}$ from the first and the third one was placed $3 \mathrm{~mm}$ from the latter. Metal crowns were made in order to reproduce a situation of interdental contact points of 2 and $3 \mathrm{~mm}$ (bone crest-contact point). Clinical assessments were performed 12 weeks after the installation of the prosthesis to verify the distance of the spaces found between the gingival papillae and the contact point. Then, the mean values obtained were subjected to statistical analysis ( 2 way ANOVA and Bonferroni post-hoc). A $\mathrm{p}<0.05$ was considered as statistically significant.

Results: the larger space was found in the situation in which the distance between implants was $2 \mathrm{~mm}$ and height of the contact point of $5 \mathrm{~mm}$. The lowest value was found in the situation in which the distance between implants was $2 \mathrm{~mm}$ and the height of the contact point of $3 \mathrm{~mm}$.

Conclusion: three mm contact points positively influence the formation of gingival papillae.
\end{abstract}

Key words: Dental papilla, Bone resorption, Dental implants, Dental prosthesis.

Recibido para publicación: julio 10 de 2013. Aceptado para publicación: noviembre 23 de 2013. 


\section{INTRODUCCIÓN}

Los implantes dentales, en especial aquellos colocados en la región anterior, además de devolver función deben ofrecer una estética favorable. Uno de los factores más importantes para el éxito del tratamiento estético es la preservación de la arquitectura de los tejidos blandos. ${ }^{1-3}$

Las papilas interproximales son de inmensa preocupación para los periodoncistas y rehabilitadores orales puesto que su pérdida puede llevar a deformidades estéticas, dificultades en la fonación e impactación alimentaria. ${ }^{4}$ Siendo así, el éxito de la rehabilitación oral con implantes dentales depende en un alto grado de la integración entre el sistema de implantes con los componentes protésicos y tejidos bucales duros y blandos.

Frecuentemente, la pérdida de la cresta ósea se observa durante el primer año en que el implante es colocado en función, seguida de una mínima pérdida ósea menor o igual a 0,2 $\mathrm{mm}$ anualmente. Esta pérdida ósea alrededor de los implantes, según su magnitud, puede interferir en los resultados estéticos una vez concluido el tratamiento restaurador ya que según estudios clínicos, la altura de la cresta ósea tiene influencia en la presencia y altura de papilas interproximales. ${ }^{5}$

Algunos de los posibles factores etiológicos que producen pérdida ósea son la anatomía y el tratamiento de superficie del implante en la región de cresta ósea, el trauma quirúrgico, la sobrecarga oclusal, la periimplantitis y el espacio biológico no respetado.,7

El espacio biológico en dientes naturales se ha estudiado ampliamente, de la misma forma este espacio es de extrema importancia en la rehabilitación con implantes. Cuando el plan de tratamiento involucra la colocación de implantes dentales en dos fases, estas estructuras se definen después de la segunda abertura quirúrgica, en cambio cuando los implantes son planeados para recibir carga inmediata, la conformación de los tejidos blandos comienza en el primer estadio quirúrgico, al momento de colocar la corona provisional. $^{8}$

Tarnow y colaboradores (1992) analizaron diferentes distancias verticales entre la base del punto de contacto interproximal y la cresta ósea; cuando la distancia era de $5 \mathrm{~mm}$ o menor, la papila estaba presente en $98 \%$ de los casos. Cuando la distancia era de $6 \mathrm{~mm}$, la papila se formó favorablemente en $56 \%$ de los casos y si la distancia era de $7 \mathrm{~mm}$ o mayor, hubo formación de papila solo en $27 \%$ de los casos. ${ }^{9}$

Además de la distancia vertical, la pérdida ósea alrededor de los implantes también está influenciada por la distancia horizontal entre implantes conti- guos. ${ }^{4,10,11}$ Un estudio analizó radiografías de 36 pacientes que presentaban dos implantes contiguos, la pérdida ósea lateral fue medida de la cresta ósea a la superficie del implante. Los resultados demostraron que la pérdida de la cresta ósea en implantes con una distancia mayor o igual a $3 \mathrm{~mm}$ fue menor que en los implantes con distancias menores a $3 \mathrm{~mm} .{ }^{4}$ Los mismos autores también sugieren el uso de implantes de menor diámetro con el propósito de mejorar la estética, principalmente en la región anterior.

A pesar de que son muchas las variables que influyen e interfieren en la obtención de una estética favorable en la terapia con implantes, este estudio "In vivo" tuvo como objetivo verificar en un mismo huésped la influencia de diferentes distancias verticales (cresta - punto de contacto) y horizontales (distancia entre implantes) en la formación de las papilas interproximales.

\section{MATERIALES Y MÉTODOS}

Este trabajo fue aprobado por la Comisión de Ética en Investigación con Animales (CEUA) de la Universidad de São Paulo (USP), protocolo No 05.1.1318.53.1. Para el experimento fueron seleccionados ocho perros adultos jóvenes de sexo masculino, con un peso aproximado de $25 \mathrm{Kg}$, en buen estado de salud y sin presencia de lesiones orales de naturaleza viral o fúnguica.

Los animales, provenientes del Biotério de la Facultad de Odontología de Ribeirao Preto de la USP, fueron mantenidos en cuarentena para la aplicación de todas las vacunas y vitaminas necesarias para que pudieran adquirir condiciones físicas óptimas para la ejecución del experimento. Durante todo el periodo experimental los animales recibieron una dieta balanceada, libre acceso a agua y control de las condiciones periodontales.

La ejecución de este trabajo fue dividida en cinco fases (Figura 1) de las cuales dos eran quirúrgicas y tres protésicas. Durante todo el experimento los animales fueron monitoreados por un veterinario.

\section{Fase I quirúrgica (primera semana)}

Antes de la primera cirugía, los animales fueron sometidos a una profilaxis con ultra sonido (Cavitron Select 3000, Dentisply MFG, Co, EE.UU.) para la remoción de biofilm bacteriano supragingival. Posteriormente, recibieron una inyección intramuscular de pre anestésico Rompun al 2\% (20 mg/Kg. Laboratório Bayer, Porto Alegre, RS, Brasil). En seguida fue realizada la inducción anestésica con una inyección intravenosa de Thiopental sódico (12,5 mg/Kg diluido en $50 \mathrm{~mL}$ de solución salina, Laboratório Cristália, Itapira, SP, Brasil). 


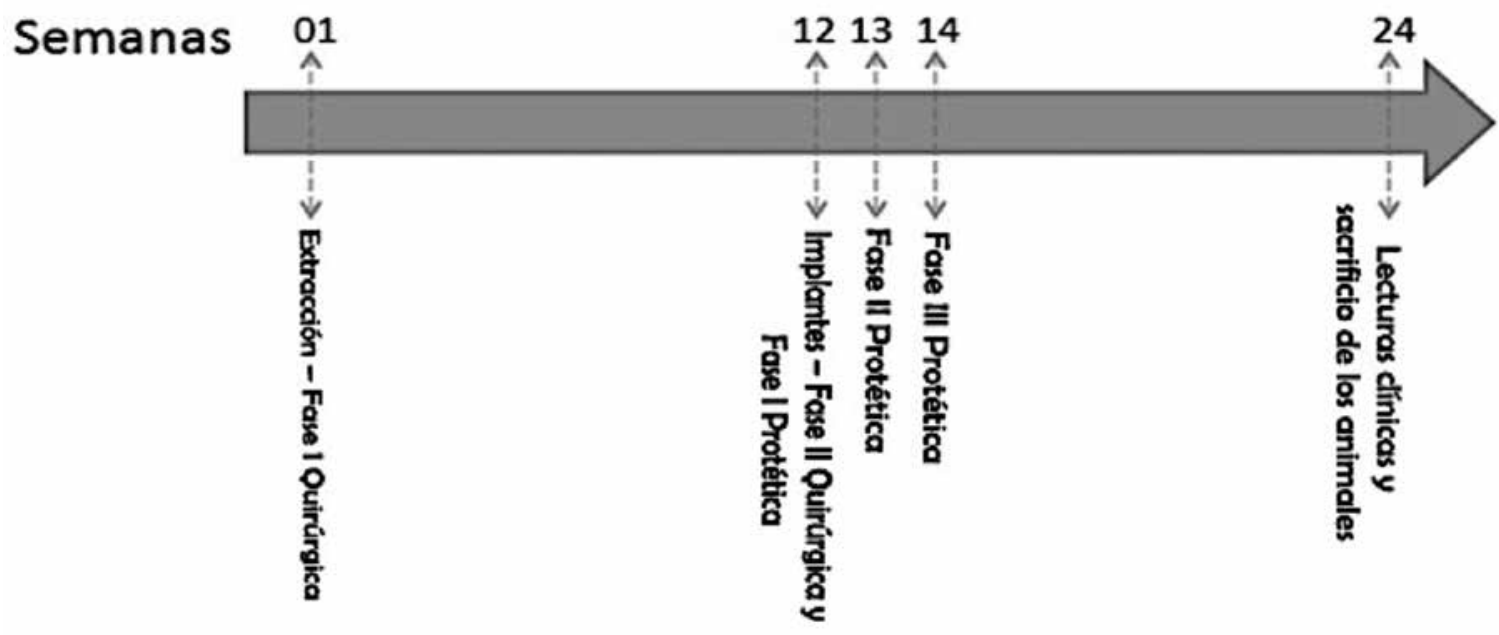

Figura 1. Cronograma del desarrollo del experimento.

Luego de la aplicación del anestésico local, (dos cárpulas de Cloridrato de Mepivacaina $20 \mathrm{mg} / \mathrm{mL}$ con epinefrina $0,01 \mathrm{mg} / \mathrm{mL}$, Dentsply Pharmaceutical, Mepivalem $A D$ ) fue levantado un colgajo total en la región de los cuatro premolares mandibulares: p1, p2, p3, p4 de cada hemiarcada mandibular (Figura 2).

Los dientes (p2i, p2d, p3i, p3d, p4i, p4d) fueron seccionados en sentido vestíbulo-lingual para evitar pérdida ósea durante las extracciones. Los colgajos fueron reposicionados y suturados con hilo reabsorbible 4-0 (Johnson \& Johnson, São José dos Campos, SP, Brasil) y se esperó un periodo de cicatrización de tres meses.

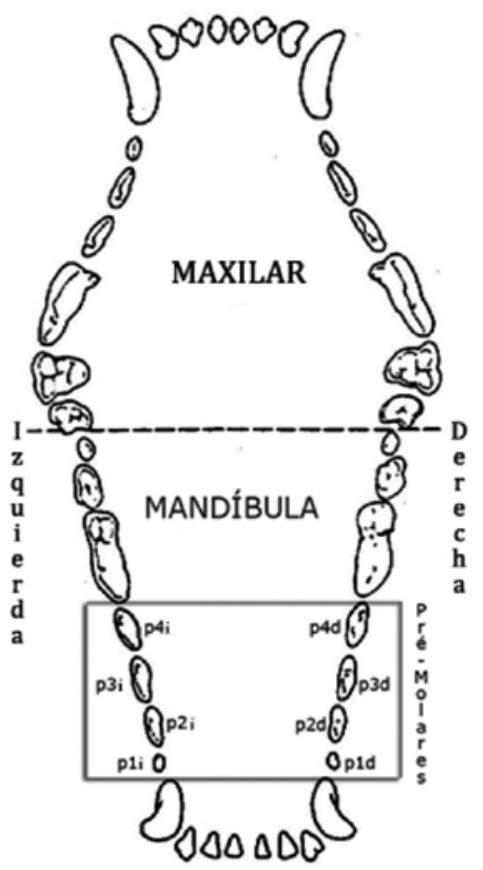

Figura 2. Esquema de la región de los cuatro premolares mandibulares izquierdos y derechos que fueron extraídos.

\section{Fase II quirúrgica (semana 12)}

Después de doce semanas, los animales fueron preparados para la segunda fase quirúrgica; recibieron una inyección intramuscular de antibiótico (20.000 U.I. de penicilina y estreptomicina 1,0 g/10 Kg, Pentabiótico Veterinário de Pequeno Porte. Wyeth Laboratory, São Bernardo do Campo, SP, Brasil) y la dosis fue repetida después de cuatro días para dar mayor cobertura antibiótica. ${ }^{12}$

Luego de repetir la técnica anestésica descrita anteriormente, se realizó una incisión horizontal sobre el reborde alveolar desde distal del canino hasta mesial del primer molar (Figura 3). Al seguir las orientaciones del fabricante, fueron colocados tres implantes a nivel óseo con dimensiones de $4,3 \mathrm{~mm}$ de diámetro y $10 \mathrm{~mm}$ de largo en cada hemiarcada mandibular (Replace Select Tapered, Nobel Biocare AB, Gotemburgo, Suecia) (Figura 4).

El primer implante fue colocado a $10 \mathrm{~mm}$ distal del canino remanente, el segundo (intermedio) fue colocado a $2 \mathrm{~mm}$ del primero y el tercer implante fue colocado a $3 \mathrm{~mm}$ del segundo (Figura 4). Las distancias entre los implantes fueron repetidas en el lado opuesto, sin embargo se varió la posición entre ellos para respetar el método de aleatorización "cross-location" (Figura 5).

\section{Fase I protésica (semana 12)}

Los implantes fueron moldeados para la confección de las prótesis definitivas. Se utilizaron cubetas parciales metálicas perforadas y material de impresión de alta precisión (Xantopren \& Optosil, Heraeus Kulzer GmbH, Hanau, Germany), con los transferentes correctamente posicionados (Figura 6). 


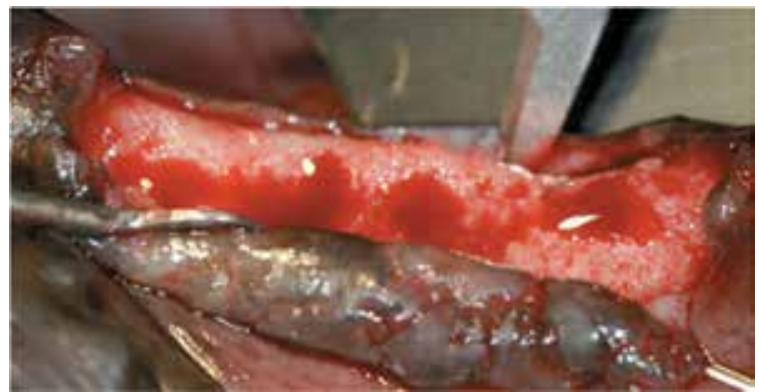

Figura 3. Incisión sobre el reborde alveolar.

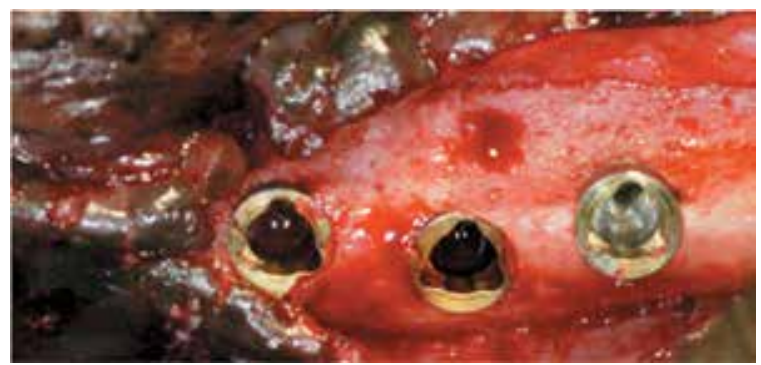

Figura 4. Vista oclusal de los tres implantes posicionados.

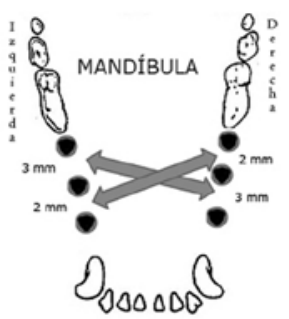

Figura 5. Localización y distancia entre los implantes.

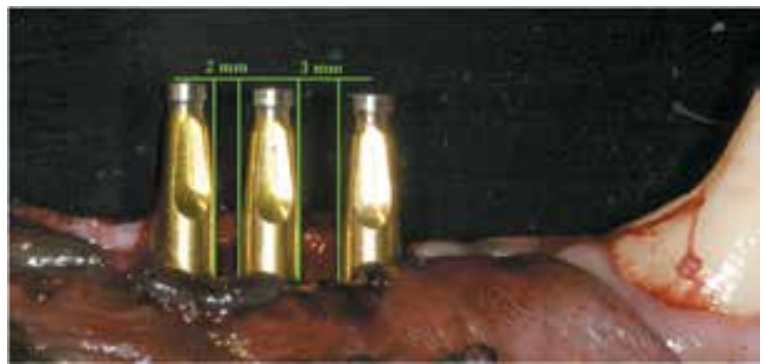

Figura 6. Transferentes en posición, se observa el correcto paralelismo y distancia entre los implantes

\section{Fase II y III Protética: (semanas 13 y 14)}

En este periodo, los cicatrizadores fueron removidos y se colocaron coronas definitivas de niquel-cromo. Estas coronas presentaban orificios de retención en proximal y se unieron con resina acrílica autopolimerizable Duralay ${ }^{\circledR}$ (Reliance Dental Mfg. Co, Worth, USA) sin alterar la altura de los puntos de contacto (Figura 7).

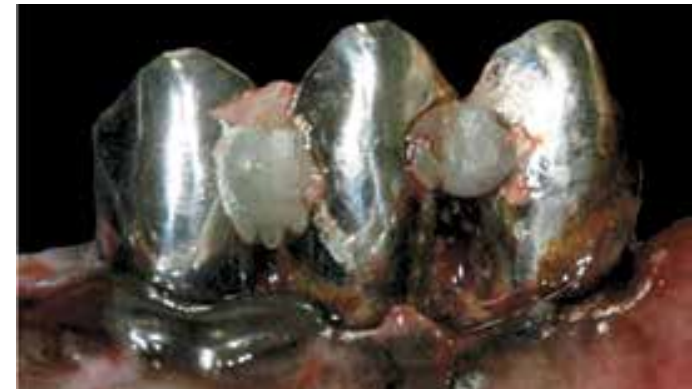

Figura 7. Coronas metálicas definitivas ferulizadas con resina acrílica.

\section{Lecturas clínicas y sacrificio (semana 24)}

Se realizaron profilaxis semanalmente con puntas de ultrasónido durante todo el periodo de permanencia de las prótesis hasta el sacrificio de los animales. A la décima semana de la colocación de las prótesis definitivas, los animales fueron sedados para realizar las lecturas clínicas y evaluar la formación de papilas.

Para determinar la altura de las papilas interproximales, con ayuda de un compás de punta seca, se trazaron medidas del espacio comprendido entre la punta de la papila hasta el punto de contacto, tales medidas fueron substraídas del valor original de la distancia del punto de contacto a la cresta ósea (3 $\mathrm{mm}$ o $5 \mathrm{~mm}$ ). Siendo así, valores mayores del espacio papila-punto de contacto estaban relacionados con una menor altura de papila interproximal.

El delineamiento experimental fue ejecutado llevando en consideración las diferentes distancias verticales y horizontales, así, fueron analizadas ocho situaciones representadas en las figuras 8 y 9 y en la Tabla 1. Después de todas las evaluaciones clínicas, los animales fueron sacrificados con una dosis letal de Thiopental.

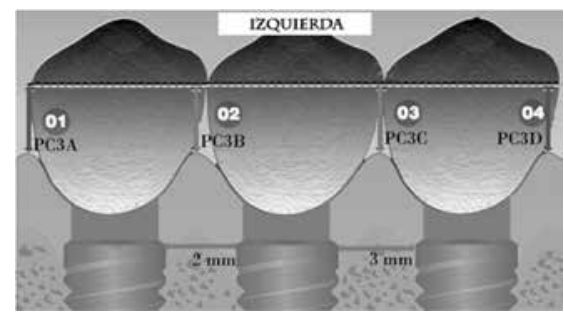

Figura 8. Distribución de las situaciones ocurridas en el lado izquierdo, donde la distancia del punto de contacto a la cresta ósea era de $3 \mathrm{~mm}$.

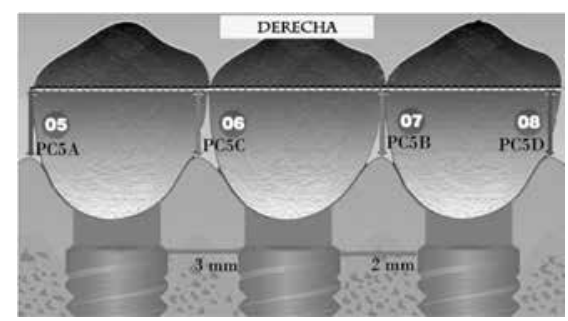

Figura 9. Distribución de las situaciones ocurridas en el lado derecho, donde la distancia del punto de contacto a la cresta ósea era de $5 \mathrm{~mm}$. 
Tabla 1. Distribución de las situaciones analizadas llevando en consideración el local de lectura y la altura del punto de contacto

\begin{tabular}{lcccc}
\hline & Extremo mesial & $\begin{array}{c}\text { Distancia entre } \\
\text { implantes } \mathbf{2 ~} \mathbf{~ m m}\end{array}$ & $\begin{array}{c}\text { Distancia entre } \\
\text { implantes } \mathbf{3} \text { mm }\end{array}$ & Extremo distal \\
\hline Punto de contacto $=3 \mathrm{~mm}$ & PC3A & PC3B & PC3C & PC3D \\
Punto de contacto $=5 \mathrm{~mm}$ & PC5A & PC5B & PC5C & PC5D \\
\hline
\end{tabular}

\section{Análisis estadístico}

La variable dependiente fue la distancia entre el punto de contacto de las coronas implantosoportadas y la punta de las papilas, en milímetros.

Dos factores de variación fueron estudiados, uno de ellos relacionado con las cuatro situaciones horizontales (extremo mesial, interimplantes con $2 \mathrm{~mm}$, interimplantes con $3 \mathrm{~mm}$ y extremo distal) y el segundo factor relacionado con las distancias verticales ( 3 y $5 \mathrm{~mm}$ ). Por lo tanto, ocho situaciones fueron obtenidas y cada uno de los perros fue parte de todas las situaciones, caracterizando un pareamiento estadístico.

Fue utilizado el Análisis de Varianza ANOVA de dos factores con un test complementario de Bonferroni para identificar las diferencias. El nivel de significancia utilizado fue de $0,05(5 \%)$ y los datos fueron analizados con el programa SPSS for Windows - Versión 15.0.0, 2006 (SPSS Inc., Chicago, Ill, EUA).

\section{RESULTADOS}

Después del periodo de cicatrización de 32 semanas se observó un índice de éxito de $100 \%$, es decir, todos los implantes se mantuvieron estables sin ninguna señal de infección hasta el final del experimento. Una vez realizado el registro de los datos clínicos en los ocho animales, fueron obtenidos los promedios del espacio entre la punta de papila y el punto de contacto para cada una de las diferentes situaciones (Tabla 2).

El mayor espacio fue encontrado en la situación seis (distancia entre implantes de $2 \mathrm{~mm}$ y altura del punto de contacto de $5 \mathrm{~mm}$ ) (PC5B). El menor valor fue encontrado en la situación tres (distancia entre implantes $2 \mathrm{~mm}$ y altura del punto de contacto de $3 \mathrm{~mm}$ ) (PC3C) (Figura 10). En la Tabla 3 se observan los valores obtenidos mediante la prueba ANOVA de dos vías y el test de Bonferroni.

El análisis aislado de los resultados estadísticos del lado izquierdo (punto de contacto de $3 \mathrm{~mm}$ ) mostró una diferencia estadísticamente significativa entre la situación de extremo mesial (PC3A) y las situaciones PC3B y PC3C. Ya en el lado derecho (punto de contacto de $5 \mathrm{~mm}$ ) hubo diferencia estadísticamente significativa entre la situación de extremo mesial (PC5A) y la situación PC5B. Las demás situaciones no presentaron una diferencia estadísticamente significativa.

Cuando fueron comparadas las situaciones de ambos lados (lado derecho vs lado izquierdo), todos los casos presentaron una diferencia estadísticamente significativa, excepto la situación de extremo mesial (Tabla 3).

Tabla 2. Valores promedio de las diferentes situaciones clínicas (distancia entre la papila y el punto de contacto).

\begin{tabular}{ccccccccc}
\hline Situaciones & PC3A & PC3B & PC3C & PC3D & PC5A & PC5B & PC5C & PC5D \\
\hline Valores promedio $(\mathrm{mm})$ & 2,9 & 1,8 & 2,0 & 2,5 & 3,0 & 3,9 & 3,6 & 3,5 \\
\hline
\end{tabular}

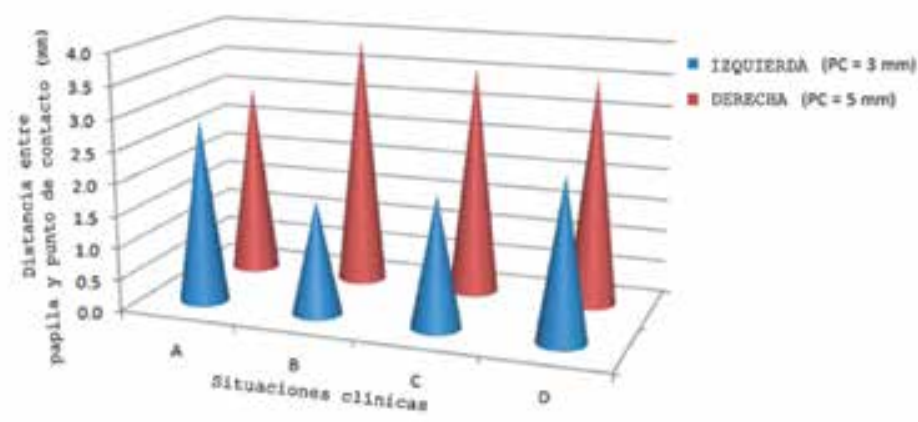

Figura 10. Gráfico ilustrativo de los promedios encontrados en las diferentes situaciones (punto de contacto - papila). $\mathrm{A}=$ Extremo mesial, $\mathrm{B}=\mathrm{Distancia}$ entre implantes de $2 \mathrm{~mm}, \mathrm{C}=$ Distancia entre implantes de $3 \mathrm{~mm}$ y $\mathrm{D}=$ Extremo distal. 
Tabla 3. Análisis estadístico de los valores encontrados entre la papila y punto de contacto (en $\mathrm{mm} \pm$ desviación estándar).

\begin{tabular}{ccccc}
\hline Altura Papila & A & B & C & D \\
\hline $3 \mathrm{~mm}$ & $2,9 \pm 0,5 \mathrm{Aa}$ & $1,8 \pm 0,6 \mathrm{Ba}$ & $2,0 \pm 0,8 \mathrm{Ba}$ & $2,5 \pm 0,5 \mathrm{Aba}$ \\
$5 \mathrm{~mm}$ & $3,0 \pm 0,5 \mathrm{Aa}$ & $3,9 \pm 0,7 \mathrm{Bb}$ & $3,6 \pm 0,4 \mathrm{ABb}$ & $3,5 \pm 0,4 \mathrm{ABb}$ \\
\hline
\end{tabular}

Letras diferentes mayúsculas en sentido horizontal y minúsculas en sentido vertical representan diferencia estadísticamente significativa $(\mathrm{p}<0,05)$. Letras iguales representan semejanza estadística.

\section{DISCUSIÓN}

A pesar de que muchos autores evalúan el índice de éxito en la terapia con implantes al tener en consideración apenas el tiempo de sobrevivencia de los mismos, ${ }^{13-16}$ los resultados de este trabajo coinciden con los de Hermann y colaboradores (1997) quienes consideran que el éxito de los implantes depende de su integración con los tejidos duros y blandos. ${ }^{17}$

Una evaluación radiográfica realizada por Tarnow y colaboradores (2000) reveló que cuando la distancia entre los implantes era mayor o igual a $3 \mathrm{~mm}$, la cresta ósea se mantenía y que cuando los implantes eran separados por distancias menores a $3 \mathrm{~mm}$, la región de la cresta ósea presentaba reabsorciones debido a la pérdida ósea lateral que ocurría alrededor de los implantes ${ }^{4}$ por tanto, las diferentes distancias entre implantes evaluadas influyen en la preservación y formación de la papila interdental entre las prótesis sobre implantes. Estas evidencias no concuerdan con los resultados encontrados en el presente estudio en perros, donde distancias de $2 \mathrm{y}$ $3 \mathrm{~mm}$ entre implantes no produjeron una diferencia estadísticamente significativa en la formación de las papilas. Estos hallazgos pueden ser justificados debido al hecho de que el reborde alveolar de estos animales es considerablemente más denso que el reborde alveolar humano, de esta manera, al ocurrir una pérdida ósea en estos rebordes alveolares densos, el compromiso óseo en dirección lateral no es suficiente como para afectar significativamente la región de la cresta alveolar.

Otros estudios realizados en perros han afirmado que distancias de 2 y $3 \mathrm{~mm}$ entre implantes no generan una diferencia significantiva en la formación de papilas interproximales y corroboran los resultados encontrados en este trabajo. ${ }^{18,19}$

Este estudio también está de acuerdo con el trabajo de Tarnow y colaboradores (1992), quienes evaluaron en seres humanos la distancia de la base del punto de contacto a la cresta ósea entre dos dientes y determinaron que idealmente las prótesis sobre implantes deben ser planeadas al promover una distancia igual o menor de $5 \mathrm{~mm}$, nunca mayor. ${ }^{9}$ De igual manera, en el presente estudio clínico en perros también se encontró que el mejor cuadro clínico (mayor formación de papila) fue observado cuando la distancia del punto de contacto a la cresta ósea fue de $3 \mathrm{~mm}$.

Este estudio fue realizado con la intención de aclarar algunas dudas aún presentes entre profesionales rehabilitadores y demostrar la importancia de una buena planeación protésica. El objetivo fue alcanzado puesto que fue posible observar la respuesta de los tejidos blandos frente a diferentes situaciones clínicas que pueden presentarse en nuestros pacientes. Sin embargo, se considera necesario realizar más estudios semejantes que evalúen estas condiciones en un mayor lapso de tiempo, de esa forma se podrían observar y evaluar reabsorciones óseas de mayor magnitud y el impacto de estas en la formación de las papilas interdentales.

\section{Conclusión}

La presencia de un punto de contacto de $3 \mathrm{~mm}$ influye positivamente en la formación de las papilas interdentales.

\section{BIBLIOGRAFÍA}

1. Albrektsson T, Dahl E, Enbom L, Engevall S, Engquist B, Eriksson AR, et al. Osseointegrated oral implants. A Swedish multicenter study of 3139 consecutively inserted Nobelpharma implants. J Periodontol 1988; 59: 287 - 296.

2. Choquet V, Hermans M, Adriaenssens P, Daelemans P, Tarnow DP, Malevez C. Clinical and radiographic evaluation of the papilla level adjacent to single-tooth dental implants. A retrospective study in the maxillary anterior region. J Periodontol 2001; 72: 1364 - 1371.

3. Belser U, Buser D, Higginbottom F. Consensus statements and recommended clinical procedures regarding esthetics in implant dentistry. Int J Oral Maxillofac Implants 2004; 19 Suppl: $73-74$.

4. Tarnow DP, Cho SC, Wallace SS. The effect of inter-implant distance on the height of inter-implant bone crest. J Periodontol 2000; 71: 546 - 549 .

5. Cappiello M, Luongo R, Di Lorio D, Bugea C, Cocchetto R, Celletti R. Evaluation of peri-implant bone loss around platform-switched implants. Int J Periodontics Restorative Dent 2008; 28: 347 - 355.

6. Oh TJ, Yoon J, Mishc CE, Wang HL. The causes of early implant bone loss: Myth or since? J Periodontol 2002; 73: $322-333$.

7. Morris HF, Ochi S, Crum P, Orenstein IH, Winkler S. AICRG, part I: a 6-year multicentered, multidisciplinary clinical study of a new and innovative implant design. J Oral Implantol 2004; 30: $125-133$. 


\section{ARTÍCULO DE INVESTIGACIÓN CIENTÍFICA Y TECNOLÓGICA}

8. Buser D, Weber HP, Donath K, Fiorelline JP, Paquette DW, Williams RC. Soft tissue reactions to nom-submerged unloaded titanium implants in beagle dogs. J Periodontol 1992; 63: 226 - 236.

9. Tarnow DP, Magner AW, Flecther P. The effect of the distance from the contact point to the crest of bone on the presence or absence of the interproximal dental papilla. J Periodontol 1992; 63: 995 - 996.

10. Gastaldo JF, Cury PR, Sendyk R. Effect of the vertical and horizontal distances between adjacent implants and between a tooth and implants on the incidence of interproximal papilla. J Periodontol 2004; 75: 1242 - 1246.

11. Scarano A, Assenza B, Piattelli M, Thams U, Roman Fs, Favero GA. Interimplant distance and crestal bone resorption: a histologic study in the canine mandible. Clin Implant Dent Related Res 2004; 6: 150 - 156.

12. Novaes Jr AB, Vidigal Jr GM, Novaes AB, Grisi MFM, Polloni S, Rosa A. Immediate implants placed into infected sites: A histomorfometric study in dogs. Int J Oral Maxillofac Implants 1998; 13: $113-118$.

13. Adell R, Eriksson B, Lekholm U, Branemark PI, Jemt T. Long-term follow-up study of osseointegrated implants in the treatment of totally edentulous jaws. Int J Oral Maxillofac Implants 1990; 5: 347 - 349.

14. Schwartz-Arad D, Chaushu, G. Placement of implants into fresh extraction sites: 4 to 7 years retrospective evaluation of 95 immediate implants. J Periodontol 1997; 68: $1110-1116$.

15. Friberg B, Jemt $\mathrm{T}$, Lekholm U. Early failures in 4,641 consecutively placed Branemark dental implants: a study from stage 1 surgery to the connection of completed prostheses. Int. J Oral Maxillofac Implants 1991; 6: 142 - 146.

16. Silverstein L, Garg A, Callan D, Shatz P. The key to success: maintaining the long-term health of implants. Dent Today 1998; 17: $104-111$.

17. Hermann JS, Cochran DL, Nummikoski PV, Buser D. Crestal bone changes around titanium implants. A radiographic evaluation of unloaded non-sumerged implants in the canine mandible. J Periodontol 1997; 68: 1117 - 1130.

18. Papalexiou V, Novaes Jr, AB, Ribeiro RF, Muglia VA, Oliveira RR. Influence of the interimplant distance on crestal bone resorption and bone density: A histomorphometric study in dogs. J Periodontol 2006; 77: $614-621$.

19. Novaes Jr, AB, Barros RRM, Muglia VA, Borge GJ. Influence of interimplant distances and placement depth on papilla formation and crestal resorption: a clinical and radiographic study in dogs. J Oral Implantol 2009; 35: $18-27$.

Correos electrónicos de los autores:

Diogo Rodrigues Cruvinel: drcruvinel.usp@gmail.com Carla Cecilia Alandia-Román: ccalandia@usp.br Camilo Andrés Villabona López: lacho.villa@hotmail.com Valdir Antonio Muglia:mugliava@forp.usp.br

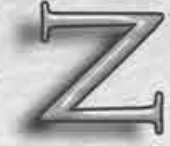

La Facultad de Odontología de la Universidad Santo Tomás en concordancia con la visión institucional aspira lograr:

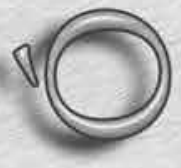

Un compromiso efectivo de la comprensión renovada del hombre colombiano, su mundo y sus valores, en un contexto de salud integral para todos; el reconocimiento nacional e internacional, como comunidad académica, a través de la investigación, la producción intelectual y el intercambio académico; liderazgo en los procesos relacionados con la salud bucal de la comunidad, a nivel regional y nacional; la cualificación permanente de sus servicios a la comunidad para responder efectiva $y$ oportunamente a sus usuarios; el fortalecimiento permanente de su accionar pedagógico mediante el desarrollo humano integral, la aplicación de tecnologias de punta y la proyección comunitaria. 